Research Paper

\title{
ABC-Transporter Expression Does Not Correlate with Response to Irinotecan in Patients with Metastatic Colorectal Cancer
}

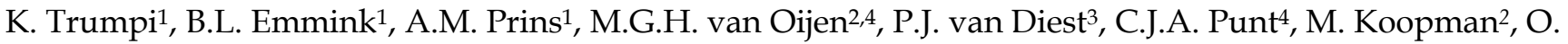 \\ Kranenburg1, I.H.M. Borel Rinkes ${ }^{1 凶}$ \\ 1. Department of Surgery, University Medical Centre Utrecht, Heidelberglaan 100, 3584CX Utrecht, The Netherlands \\ 2. Department of Medical Oncology, University Medical Centre Utrecht, Heidelberglaan 100, 3584CX Utrecht, The Netherlands \\ 3. Department of Pathology, University Medical Centre Utrecht, Heidelberglaan 100, 3584CX Utrecht, The Netherlands. \\ 4. Department of Medical Oncology, Academic Medical Centre, Meibergdreef 9, 1105AZ Amsterdam, The Netherlands \\ $\triangle$ Corresponding author: Inne HM Borel Rinkes, Department of Surgery, University Medical Centre Utrecht, Heidelberglaan 100, 3584CX Utrecht, \\ The Netherlands. Tel.: 0031-88-7558632; Fax: 0031-30-2541944; Email: i.h.m.borelrinkes@umcutrecht.nl
}

() 2015 Ivyspring International Publisher. Reproduction is permitted for personal, noncommercial use, provided that the article is in whole, unmodified, and properly cited. See http://ivyspring.com/terms for terms and conditions.

Received: 2015.05.05; Accepted: 2015.06.12; Published: 2015.09.03

\begin{abstract}
Background: Active efflux of irinotecan by ATP-binding cassette (ABC)-transporters, in particular $A B C B 1$ and $A B C G 2$, is a well-established drug resistance mechanism in vitro and in pre-clinical mouse models, but its relevance in colorectal cancer (CRC) patients is unknown. Therefore, we assessed the association between $A B C$-transporter expression and tumour response to irinotecan in patients with metastatic CRC.

Methods: Tissue microarrays of a large cohort of metastatic CRC patients treated with irinotecan in a prospective study (CAIRO study; $n=566$ ) were analysed for expression of $A B C B 1$ and $A B C G 2$ by immunohistochemistry. Kaplan-Meier and Cox proportional hazard regression analyses were performed to assess the association of $A B C$ transporter expression with irinotecan response. Gene expression profiles of 17 paired tumours were used to assess the concordance of $A B C B 1 / A B C G 2$ expression in primary $C R C$ and corresponding metastases.

Results: The response to irinotecan was not significantly different between primary tumours with positive versus negative expression of $A B C B 1$ (5.8 vs 5.7 months, $p=0.696$ ) or $A B C G 2$ (5.7 vs 6.1 months, $p=0.811$ ). Multivariate analysis showed neither $A B C B 1$ nor $A B C G 2$ were independent predictors for progression free survival. There was a mediocre to poor concordance between $A B C$-transporter expression in paired tumours.

Conclusion: In metastatic CRC, $A B C$-transporter expression in the primary tumour does not predict irinotecan response.
\end{abstract}

Key words: metastatic colorectal cancer, ABC-transporters, ABCB1, ABCG2, Irinotecan, chemotherapy, CAIRO

\section{Introduction}

The vast majority of patients with metastatic colorectal cancer (CRC) receive systemic therapy, either alone or in combination with surgery. Drugs with proven efficacy are fluoropyrimidines, oxaliplatin and irinotecan.[1] Irinotecan is effective both when given as monotherapy and in combination schedules. However, intrinsic and acquired resistance is usually observed. Interestingly, tumours progressing on fluorpyrimidine-oxaliplatin treatment can still respond to irinotecan and vice versa, suggesting different mechanisms of drug resistance.[1-3] Acquisition of 'multi-drug resistance' eventually causes failure of systemic cancer treatment.

The active efflux of chemotherapeutic agents 
such as irinotecan from tumour cells by transmembrane pumps belonging to the ATP-binding cassette (ABC) family has been extensively reported as a potential clinically relevant mechanism of (multi-)drug resistance.[4-8] ABC-transporters are expressed on differentiated cells facing the lumen, both in normal colon tissue and in colorectal tumours.[4, 9-12] In normal colon tissue this serves to expel toxic substances into the intestinal lumen to prevent their systemic uptake. Similarly, in colorectal tumours ABC-transporters are expressed on the surface of lumen-facing differentiated tumour cells.[4] ABC-transporters may thus be potential targets for reversing efflux-mediated chemotherapy resistance. However, the results of clinical trials evaluating combination therapy of ABC-transporter inhibitors and chemotherapy were negative.[13, 14] Still, high ABC-transporter expression has been associated with poor outcome.[15, 16] In this study we assessed whether ABC-transporter expression was related to the response to irinotecan in patients with metastatic CRC.

\section{Methods}

\section{Patients and tissue}

Tumour tissue was obtained from patients enrolled in the CAIRO (Sequential versus combination chemotherapy with capecitabine, irinotecan, and oxaliplatin in metastatic CRC (CAIRO): a phase III randomized controlled trial) study [17] of the Dutch Colorectal Cancer Group (DCCG). For this study formalin-fixed paraffin embedded material from the primary tumour was obtained from 566 of the 803 participating patients of the CAIRO study. No tissue material was obtained in 247 patients, because resection of the primary tumour was not performed or the material was insufficient or not available. Tissue micro arrays (TMAs) were produced by taking $2 \mathrm{~mm}$ punches of each specimen as described previously.[18]

The results of the CAIRO study have been published.[17] In brief, patients were randomized between sequential and combination treatment. Sequential treatment encompassed first line treatment with capecitabine, second line with irinotecan, and third line with oxaliplatin and capecitabine. Combination therapy encompassed first line treatment with capecitabine and irinotecan, and second line with capecitabine and oxaliplatin. The primary endpoint of the study was overall survival. Tumour response assessment was performed every 9 weeks by the RECIST criteria on a CT scan, and follow up was done every 3 months after the completion of treatment until death.

\section{Immunohistochemistry}

Slides were created by transverse sectioning $(4 \mu \mathrm{m})$. The paraffin-embedded slides were deparaffinated with xylene and rehydrated through a series of ethanol concentrations. Endogenous peroxidase activity was blocked by incubating in $0.3 \% \mathrm{H}_{2} \mathrm{O}_{2}$ in methanol at room temperature for half an hour, thereafter antigen retrieval was achieved by heating the slides in a citrate buffer, $\mathrm{pH}$ 6.0, for 20 minutes; followed by cooling in the same buffer for 10 minutes. The slides were incubated with a diluted primary antibody overnight at $4^{\circ} \mathrm{C}$. Incubation with an undiluted secondary antibody (Brightvision ${ }^{\circledR}$ ) for 30 minutes followed. Rinsing between steps was performed with phosphate buffered saline. The slides were developed with diaminobenzidine and counterstained with Bayers' haematoxylin. Hereafter the slides were dehydrated and mounted with cover slips.

For ABCG2, we used a three-step protocol as earlier described by Maliepaard et al [11], where the slides were incubated with $5 \%$ normal rabbit serum/PBS for 30 minutes prior to the incubation of the primary antibody. The slides were incubated with biotinylated rabbit anti-mouse IgG (Dako, 1:200 in $90 \%$ PBS/BSA/azide and 10\% human serum) for 30 minutes, washed, and then incubated with HRP-conjugated streptavidin (Dako, 1:1000 with additional $10 \%$ normal human serum).

The following primary antibodies were used: anti-ABCB1 HPA002199 (Atlas Antibodies Sigma-Aldrich ${ }^{\circledR}$ ) and anti-ABCG2 BXP-21 (GeneTex $\left.{ }^{\circledR}\right)$

\section{Slide scoring}

Slide scoring of the CAIRO TMA was performed by three observers (AP, BE and PvD), cores were eligible for scoring if tumour cells were identified. If the slide scoring was discordant, the opinion of pathologist PvD was final. The luminal, cytoplasmic and stromal expression of ABCB1 were evaluated. For ABCG2, the luminal and cytoplasmic expression were evaluated. The luminal expression was scored in two ways, the percentage of lumina that were positively stained, and semi-quantitatively as - = no staining, $+=$ weak,$++=$ moderate and $+++=$ strong. Cytoplasmic and stromal expression were scored semi-quantitative, in which $-=$ no staining,$+=$ weak, $++=$ moderate and $+++=$ strong.

\section{Gene expression profiling}

The TMA solely contains samples of the primary CRC of the patients in the CAIRO cohort. As these patients were not eligible for resection, metastases material was not available. To determine whether predicting irinotecan response based on primary CRC $\mathrm{ABC}$-transporter expression levels in patients with 
metastatic CRC is legitimate, we assessed the concordance of $\mathrm{ABCB} 1$ and ABCG2 expression between primary CRC and their corresponding CRLM. To this end we used the gene expression profiles of $46 \mathrm{pa}-$ tients.[19] The dataset was uploaded into the R2 microarray analysis and visualization platform (http://r2.amc.nl) and analysed. We compared the expression levels of $\mathrm{ABCB} 1$ and $\mathrm{ABCG} 2$ in normal colon tissue, primary $\mathrm{CRC}$ and the corresponding liver metastases.

\section{Statistical analysis}

Patients from the CAIRO study were subdivided into two groups based on positive or negative luminal ABC-transporter immunohistochemistry staining patterns. Associations between the staining patterns of the ABC-transporters were calculated via the Kruskal-Wallis test, $\mathrm{X}^{2}$-test or the Spearman's test where appropriate. Patients without tumour cells in the $2 \mathrm{~mm}$ cores or missing cores, or patients who failed to start second line therapy with irinotecan were excluded from the following analyses.

Patient and tumour characteristics were compared using Wilcoxon's rank sum test or $\mathrm{X}^{2}$-test where appropriate. Initial treatment response was analysed by response on the first CT-scan which was estimated using Wilcoxon $\mathrm{X}^{2}$-test. Progression-free survival (PFS) for first-line therapy was calculated from the date of randomization to the first observation of disease progression or death from any cause. PFS for second line therapy was defined as the time from the failure of the first line therapy until first progression reported after the start of second-line therapy or death, whichever came first. Treatment response was analysed by PFS curves using the Kaplan-Meier method and compared with the log-rank test. Multivariate analysis was performed using a Cox proportional hazard regression model.

Gene expression levels between corresponding tumours for the cohort of 46 patients were compared using Lin's concordance correlation coefficient.

P-values less than 0.05 were considered statistically significant. All statistical analyses were performed using SPSS 20.0 software or GraphPad Prism version 6.00 for Windows (GraphPad Software, San Diego California USA, www.graphpad.com).

\section{Results}

\section{Staining patterns of $A B C B 1$ and $A B C G 2$}

$A B C B 1$ and $A B C G 2$ are transmembrane proteins with a strong apical staining pattern in normal colon tissue.[11] From the 566 tumour specimens that were resected, 539 were eligible for scoring of the IHC stainings (Figure 1). In these 539 colorectal tumours $A B C B 1$ and $A B C G 2$ were expressed on the apical side of lumen-facing differentiated tumour cells where they promote drug efflux into the lumen. Luminal ABCB1 staining was negative in 189 (35.1\%) of the primary colorectal tumours and positive in 350 $(64.9 \%)$ tumours. The positive tumours could be subdivided into strong (111; 20.6\%), moderate (134; $24.9 \%)$ and weak $(105 ; 19.5 \%)$ tumour subgroups (Figure 2A). Luminal ABCG2 staining was negative in $126(23.4 \%)$ of the primary colorectal tumours and positive in $413(76.6 \%)$ tumours. These positive tumours could be subdivided into strong (222; $41.2 \%)$, moderate $(172 ; 31.9 \%)$ and weak $(19 ; 3.5 \%)$ tumour subgroups (Figure 2B). There was a strong and significant association between the intensity and the percentage of positive luminal staining for ABCB1 $(0.772, p=<0.001)$ and ABCG2 $(0.555, p=<0.001)$ (Figure $2 \mathrm{C} \& \mathrm{D})$. However, there was no correlation between $\mathrm{ABCB} 1$ and ABCG2 staining $(\mathrm{r}=0.208 p=<0.001)$ (Figure $2 \mathrm{E}$ ).

\section{Patient and tumour characteristics}

A total of 446 patients in this study received irinotecan, either as second line monotherapy $(n=177)$ or as first line therapy in combination with capecitabine $(n=269)$, and were used for the following analyses (Figure 1). Patient characteristics including age, gender, performance status, predominant localization of metastasis, serum lactate dehydrogenase (LDH), prior adjuvant therapy, and synchronous/metachronous metastatic disease, were equally distributed between the $A B C$ transporter subgroups for the patients included in this study (Table 1).

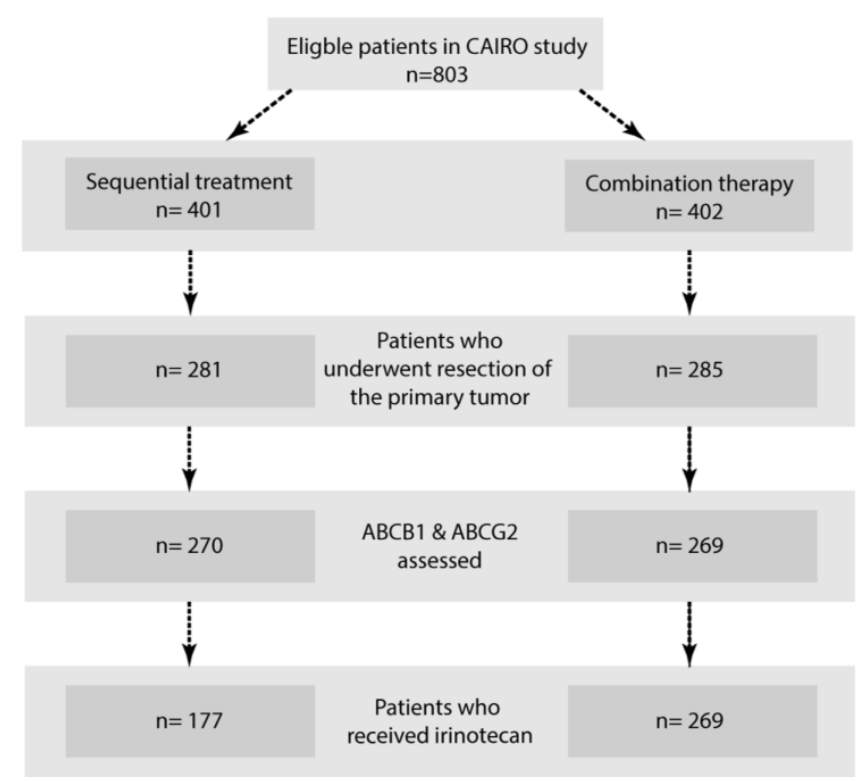

Figure 1. Flowchart. Systematic overview of included tumour specimens. 
A

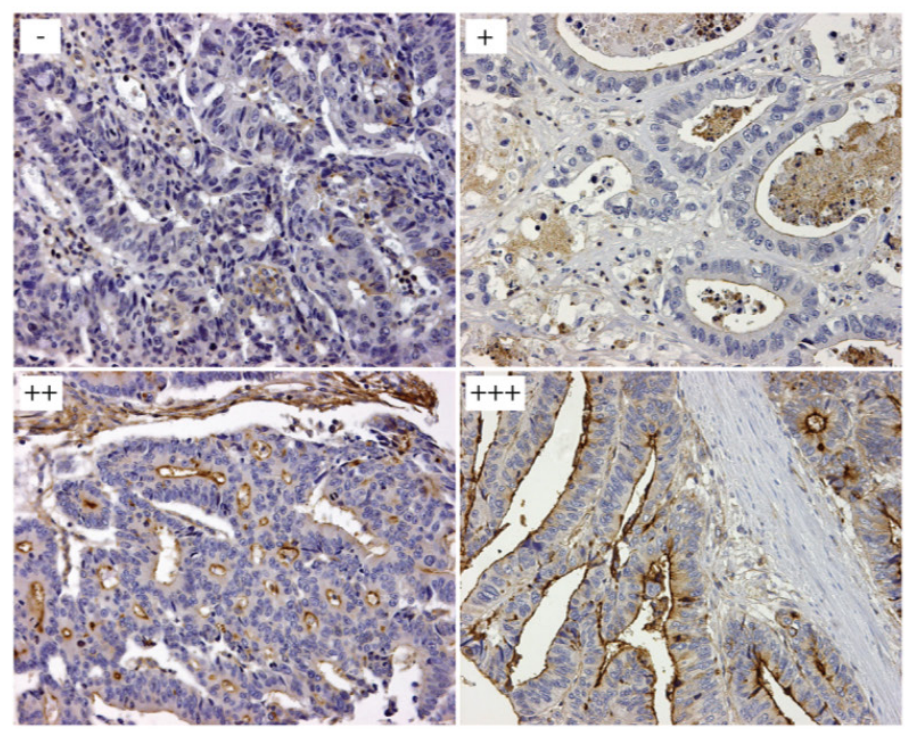

B

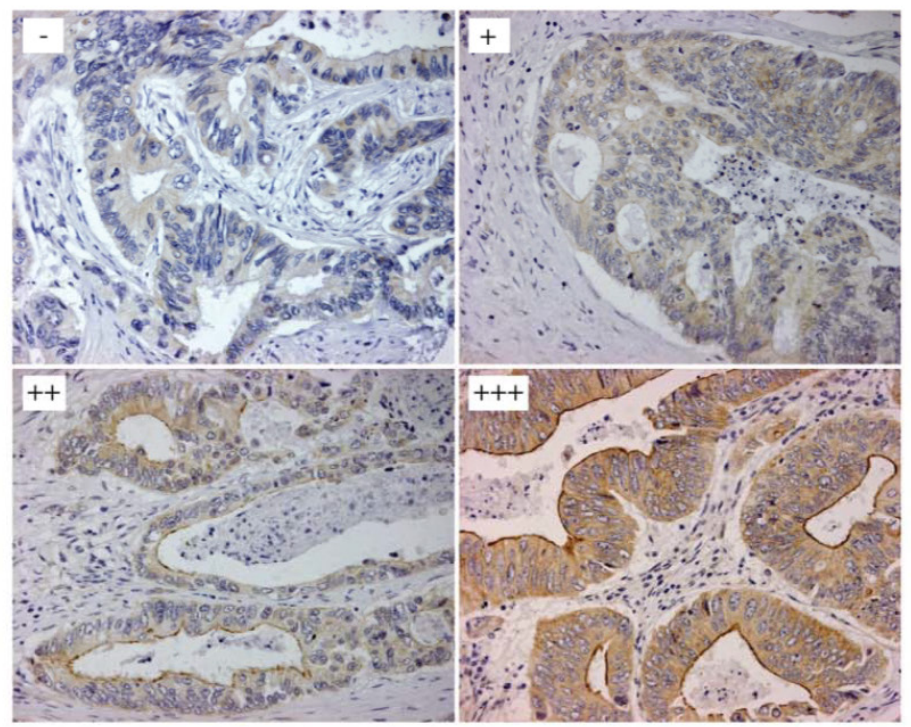

C

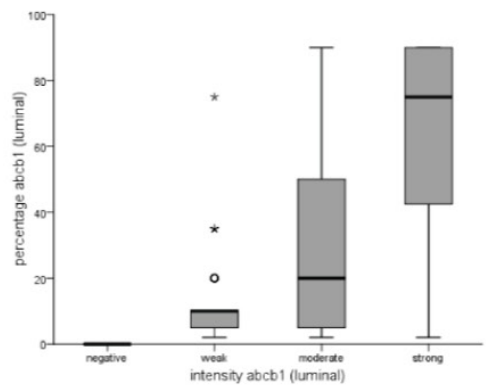

D

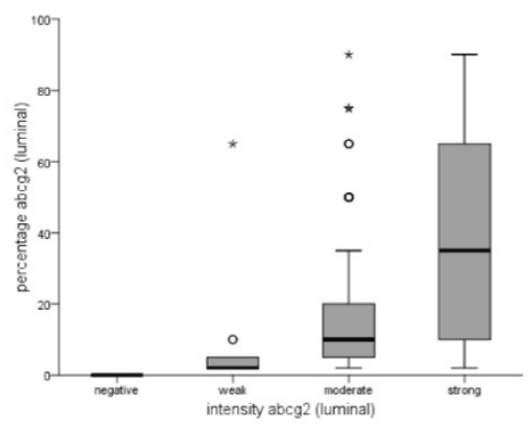

E

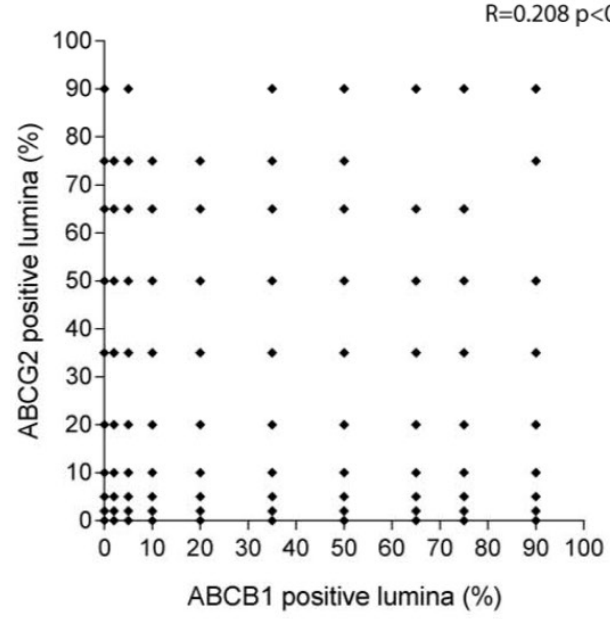

Figure 2. Luminal staining patterns of $A B C B 1$ and $A B C G 2$. A) Representative photo microscopic images of luminal $A B C B 1$ staining of formalin-fixed paraffin embedded CRC tissue (x20) with HPA002199, scored as absent (-); weak (+); moderate (++); strong (+++). B) Representative photo microscopic images of luminal $A B C G 2$ staining of formalin-fixed paraffin embedded CRC tissue (x20) with BXP-21, scored as absent (-); weak (+); moderate (++); strong $(+++)$. C) Box-Whisker plots showing strong and significant association between the intensity and the percentage of positive luminal staining for $A B C B 1$ and D) $A B C G 2$. E) No correlation was found between $A B C B 1$ and $A B C G 2$ staining $(r=0.208 p=<0.001)$ shown in this scatterplot.

ABCB1-negative tumours and ABCG2-negative tumours showed a more mucinous phenotype. ABCG2 negative tumours showed more often poor differentiation grade $(p=0.031)$ (Table 2). Furthermore, microsatellite instability (MSI) was more frequently observed in ABCB1 negative tumours $(p=0.011)$ (Table 2). There were no significant differences between the negative and positive tumours for both ABC-transporters in terms of TNM-classification and site of the primary tumour (Table 2).

\section{ABC-transporter expression does not corre- late with response to irinotecan}

There was no difference in response after the first cycle of chemotherapy between the ABCB1-positive and -negative tumours, neither for the second-line irinotecan monotherapy $(p=0.926)$, nor for the first-line capecitabine/irinotecan combination therapy $(p=0.479)$. Likewise, ABCG2-positive and -negative tumours did not show a differential initial response to either monotherapy $(p=0.879)$ or combination therapy $(\mathrm{p}=0.102)$ with irinotecan (Table $3)$. 
Table 1. Patient characteristics

\begin{tabular}{|c|c|c|c|c|c|c|c|c|c|c|}
\hline & \multicolumn{2}{|c|}{ Luminal $A B C B 1$ negative } & \multicolumn{2}{|c|}{ Luminal $\mathrm{ABCB} 1$ positive } & \multirow[t]{2}{*}{ p-value } & \multicolumn{2}{|c|}{ Luminal ABCG2 negative } & \multicolumn{2}{|c|}{ Luminal $\mathrm{ABCG} 2$ positive } & \multirow[t]{2}{*}{ p-value } \\
\hline & $n=153$ & $\%$ & $n=293$ & $\%$ & & $n=99$ & $\%$ & $n=347$ & $\%$ & \\
\hline Age & 63.2 & $(34-83)$ & 62.4 & $(31-81)$ & 0.384 & 62.3 & $(34-78)$ & 62.8 & $(31-83)$ & \\
\hline \multicolumn{11}{|l|}{ Sex } \\
\hline female & 57 & 37.3 & 103 & 35.2 & \multirow{2}{*}{0.660} & 34 & 34.3 & 126 & 36.3 & \multirow{2}{*}{0.719} \\
\hline male & 96 & 62.7 & 190 & 64.8 & & 65 & 65.7 & 221 & 63.7 & \\
\hline \multicolumn{11}{|c|}{ Performance status } \\
\hline PSO & 101 & 66.0 & 195 & 66.6 & \multirow{3}{*}{0.992} & 66 & 66.7 & 230 & 66.3 & \multirow{3}{*}{0.735} \\
\hline PS1 & 46 & 30.1 & 87 & 29.7 & & 28 & 28.3 & 105 & 30.3 & \\
\hline$\overline{P S 2}$ & 6 & 3.9 & 11 & 3.8 & & 5 & 5.1 & 12 & 3.5 & \\
\hline \multicolumn{11}{|c|}{ Predominant localization of metastases } \\
\hline liver & 108 & 70.6 & 203 & 69.3 & \multirow{3}{*}{0.901} & 62 & 62.6 & 249 & 71.8 & \multirow{3}{*}{0.067} \\
\hline extra hepatic & 44 & 28.8 & 85 & 29.0 & & 36 & 36.4 & 93 & 26.8 & \\
\hline unknown & 1 & 0.7 & 5 & 1.7 & & 1 & 1.0 & 5 & 1.4 & \\
\hline \multicolumn{11}{|l|}{$\mathrm{LDH}$} \\
\hline normal & 117 & 76.5 & 204 & 69.6 & \multirow{2}{*}{0.126} & 74 & 74.7 & 247 & 71.2 & \multirow{2}{*}{0.486} \\
\hline abnormal & 36 & 23.5 & 89 & 30.4 & & 25 & 25.3 & 100 & 28.8 & \\
\hline \multicolumn{11}{|c|}{ Prior adjuvant therapy } \\
\hline no & 126 & 82.4 & 241 & 82.3 & \multirow{2}{*}{0.979} & 76 & 76.8 & 291 & 83.9 & \multirow{2}{*}{0.103} \\
\hline \begin{tabular}{|l} 
yes \\
\end{tabular} & 27 & 17.6 & 52 & 17.7 & & 23 & 23.2 & 56 & 16.1 & \\
\hline \multicolumn{11}{|c|}{ Onset of metastasis $(6 \mathrm{~m})$} \\
\hline \begin{tabular}{|l|} 
synchronous \\
\end{tabular} & 81 & 52.9 & 134 & 46.4 & \multirow{3}{*}{0.167} & 47 & 47.5 & 168 & 48.4 & \multirow{3}{*}{0.773} \\
\hline metachronous & 71 & 46.4 & 155 & 52.9 & & 52 & 52.5 & 174 & 50.1 & \\
\hline unknown & 1 & 0.7 & 4 & 1.4 & & 0 & 0.0 & 5 & 1.4 & \\
\hline \multicolumn{11}{|l|}{\begin{tabular}{|l|} 
Treatment arm \\
\end{tabular}} \\
\hline sequential & 66 & 43.1 & 111 & 37.9 & \multirow{2}{*}{0.282} & 40 & 40.4 & 137 & 39.5 & \multirow{2}{*}{0.869} \\
\hline \begin{tabular}{|l|} 
combination \\
\end{tabular} & 87 & 56.9 & 182 & 62.1 & & 59 & 59.6 & 210 & 60.5 & \\
\hline
\end{tabular}

Age was compared via the Wilcoxon rank sum test, for all other variables the $\mathrm{X}^{2}$-test was used.

Table 2. Tumour characteristics

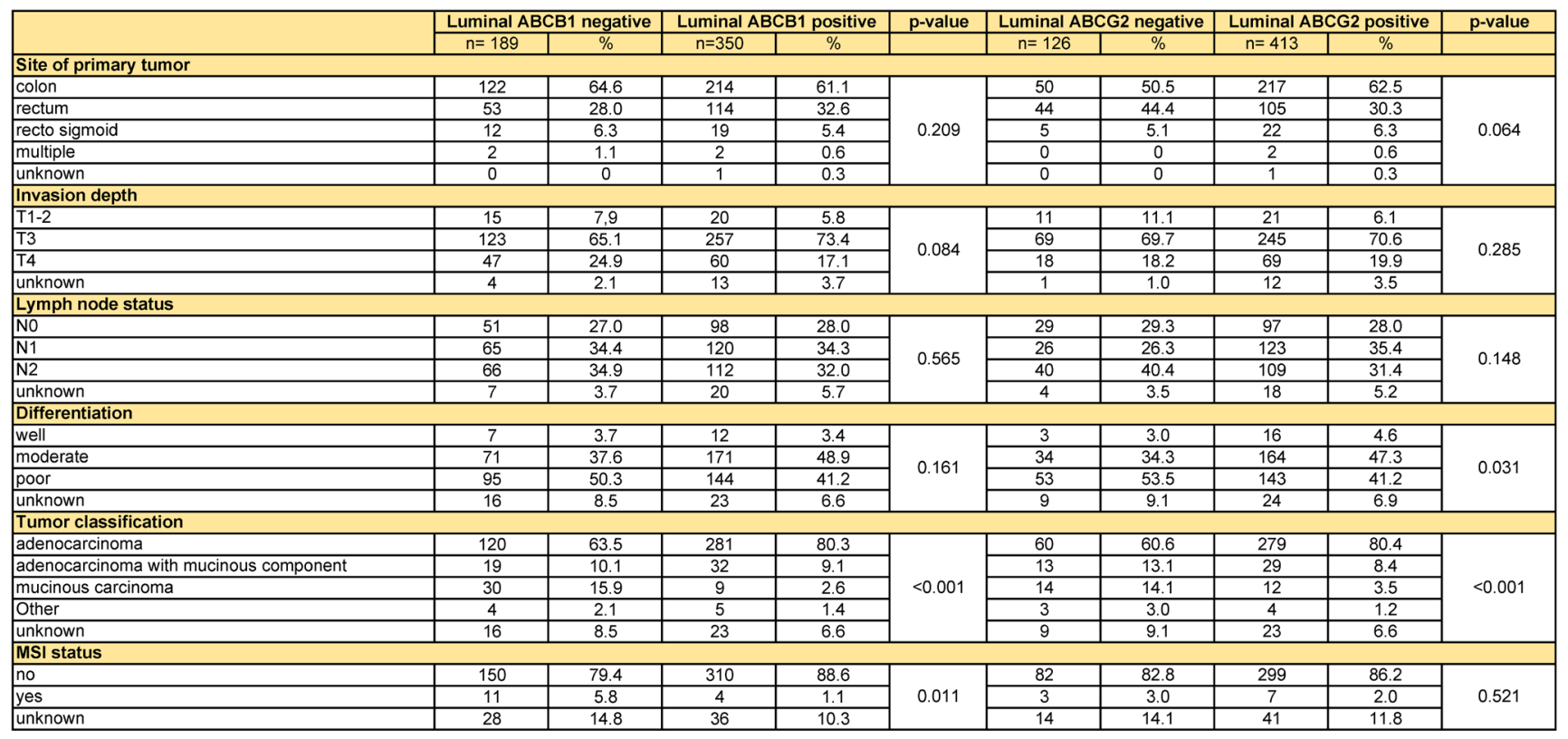

All variables were compared via the $\mathrm{X}^{2}$-test.

Table 3. Response on first cycle chemotherapy based on RECIST criteria

\begin{tabular}{|c|c|c|c|c|c|c|c|c|c|c|}
\hline \multicolumn{11}{|c|}{ CT Respons after first cycle capecitabine and irinotecan } \\
\hline & \multicolumn{2}{|c|}{ Luminal ABCB1 negative } & \multicolumn{2}{|c|}{ Luminal ABCB1 positive } & \multirow[t]{2}{*}{ p-value } & \multicolumn{2}{|c|}{ Luminal ABCG2 negative } & \multicolumn{2}{|c|}{ Luminal ABCG2 positive } & p-value \\
\hline & $n=81$ & $\%$ & $\mathrm{n}=155$ & $\%$ & & $n=54$ & $\%$ & $n=182$ & $\%$ & \multirow{6}{*}{0.102} \\
\hline Complete response & 1 & 1.1 & 0 & 0 & \multirow{5}{*}{0.479} & 1 & 1.9 & 0 & 0 & \\
\hline Partial response & 28 & 32.2 & 54 & 29.7 & & 18 & 33.3 & 64 & 35.2 & \\
\hline Stable disease & 36 & 41.4 & 79 & 43.4 & & 25 & 46.3 & 90 & 49.5 & \\
\hline Progressive disease & 13 & 14.9 & 17 & 9.3 & & 10 & 18.5 & 20 & 11.0 & \\
\hline Not available & 3 & 3.4 & 5 & 2.7 & & 0 & 0 & 8 & 4.4 & \\
\hline
\end{tabular}

\begin{tabular}{|c|c|c|c|c|c|c|c|c|c|c|}
\hline \multicolumn{11}{|c|}{ CT Respons after first cycle irinotecan } \\
\hline & Luminal & negative & Luminal & positive & $\mathrm{p}$-value & Luminal $/$ & negative & Luminal $/$ & positive & $\mathrm{p}$-value \\
\hline & $\mathrm{n}=55$ & $\%$ & $n=97$ & $\%$ & \multirow{6}{*}{0.926} & $n=33$ & $\%$ & $n=119$ & $\%$ & \multirow{6}{*}{0.879} \\
\hline Complete response & 0 & 0 & 0 & 0 & & 0 & 0 & 0 & 0 & \\
\hline Partial response & 4 & 7.3 & 7 & 7.2 & & 2 & 6.1 & 9 & 7.6 & \\
\hline Stable disease & 32 & 58.2 & 61 & 62.9 & & 21 & 63.6 & 72 & 60.5 & \\
\hline Progressive disease & 18 & 32.7 & 28 & 28.9 & & 10 & 30.3 & 36 & 30.3 & \\
\hline Not available & 1 & 1.8 & 1 & 1.0 & & 0 & 0 & 2 & 1.7 & \\
\hline
\end{tabular}


In addition, there was no significant difference in PFS between patients with tumours with luminal ABCB1 expression compared to negative tumours, neither for monotherapy of irinotecan (5.8 vs 5.7 months, $p=0.696)$, nor for combination therapy with irinotecan and capecitabine (9.7 vs 8.3 months, $p=0.153$ ) (Figure 3). Furthermore, there was no significant difference in PFS between patients with tumours with luminal ABCG2-transporter expression compared to negative tumours, in patients treated with irinotecan monotherapy (5.7 vs 6.1 months, $p=0.811$ ) and in patients treated with capecitabine with irinotecan (9.0 vs 10.0 months, $p=0.196$ ) (Figure 3).

Next, patients with completely negative tumours were compared with patients with tumours with positive staining patterns for either ABCB1 or ABCG2 or both. This did not show a significant difference in PFS, neither for the monotherapy with irinotecan ( 5.8 vs 5.6 months, $P=0.659$ ), nor for the combination therapy (9.4 vs 7.8 months, $p=0.301$ ). Finally, we compared patients with completely ABCB1/ABCG2-negative tumours patients with strong $\mathrm{ABCB} 1 / \mathrm{ABCG}$-double

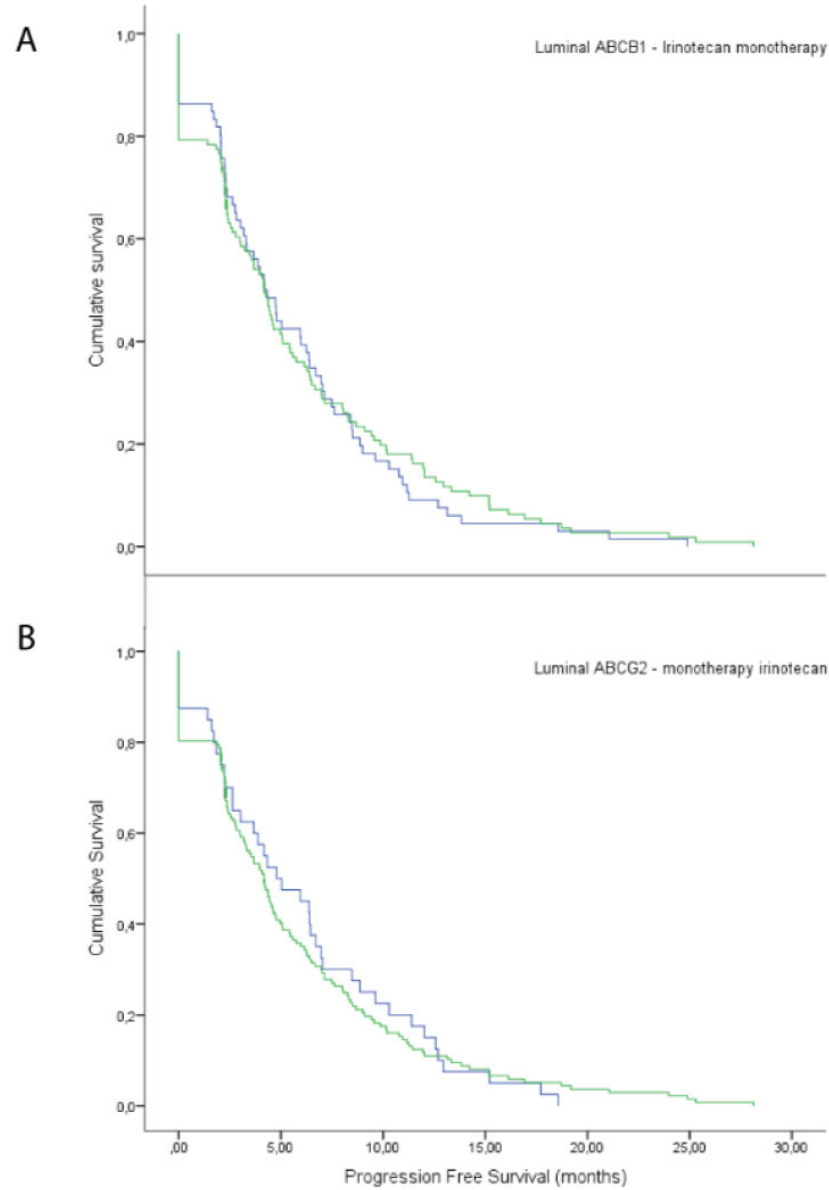

positive tumours, but also this did not yield any significant difference in PFS, neither for monotherapy $\mathrm{n}=25$ vs $\mathrm{n}=20$ ( 4.8 vs 5.6 months, $p=0.652$ ), nor for combination therapy $\mathrm{n}=29$ vs $\mathrm{n}=35 \quad(7.9$ vs 10.3 months, $p=0.458$ ).

After multivariate analysis neither ABCB1 nor ABCG2 were found to be independent predictors for PFS after monotherapy with irinotecan or combination therapy of capecitabine and irinotecan.

\section{Gene expression profiles of $A B C B 1$ and $A B C G 2$ do not correspond in primary CRC and CRLM}

In 17 paired primary CRC and CRLM [19] the Lin's concordance correlation coefficient for these paired tumours was for ABCB1 $0.545 \quad(95 \% \mathrm{CI}$ $0.125-0.809)$ and for ABCG2 $0.256 \quad(95 \% \mathrm{CI}$ -0.224-0.636). Hereby, we show a mediocre to poor correlation between ABC-transporter expression in primary CRC and their corresponding metastases (Supplemental Figure 1).

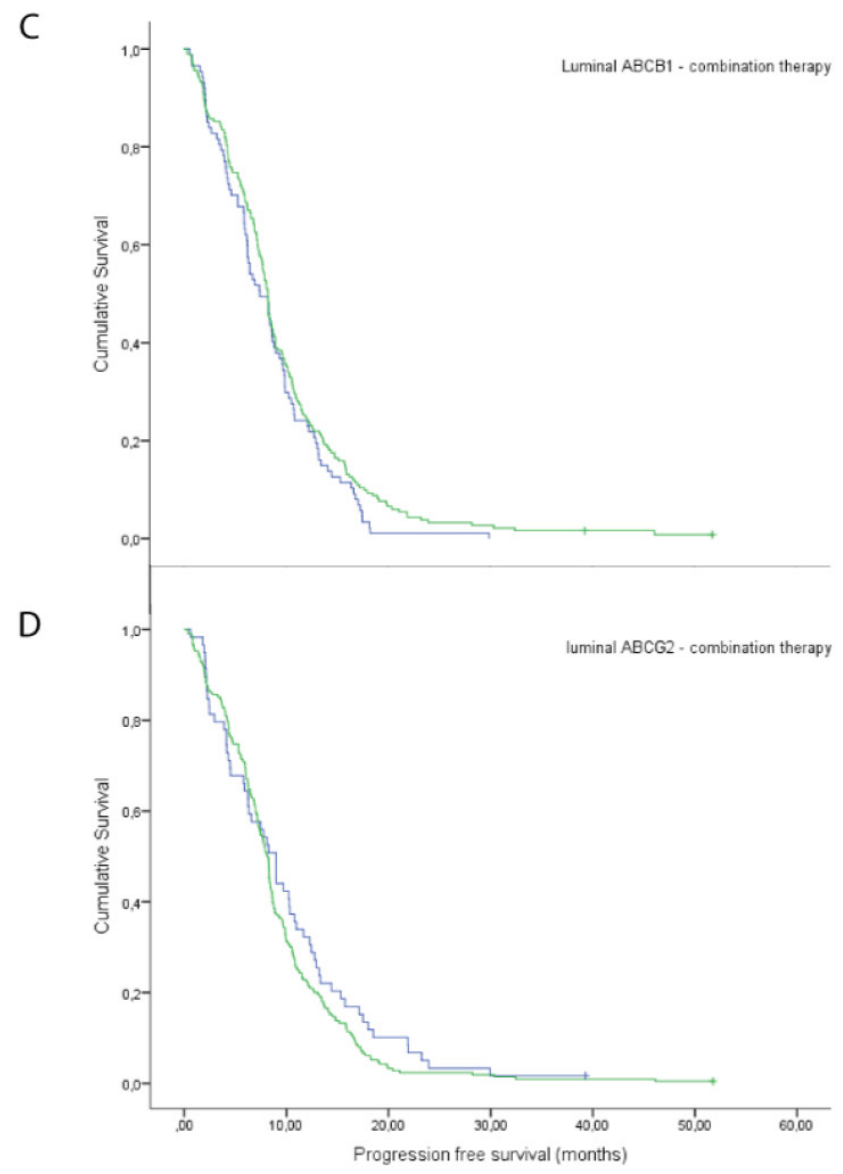

Figure 3. Response rates on irinotecan treatment. Response rates of patients with negative luminal expression versus positive, when treated with irinotecan monotherapy, $A$ ) $A B C B 1$ and $B) A B C G 2$. Response rates of patients with negative luminal expression versus positive, when treated with combination therapy of irinotecan and capecitabine, C) ABCB1 and D) ABCG2. 


\section{Discussion}

In this report we retrospectively analysed the correlation of $\mathrm{ABCB} 1$ and $\mathrm{ABCG} 2$ expression in the primary tumour with response of the corresponding metastases to irinotecan mono- and combination therapy in patients with metastatic CRC. For this purpose TMAs from the CAIRO study were used, this is the only large study cohort with tumour specimens available from CRC patients that were treated with irinotecan monotherapy. Our study shows that the response rate to irinotecan for patients with metastatic CRC is not related to expression of ABCB1 and $A B C G 2$, the two major irinotecan efflux pumps. Likewise, germ line DNA polymorphisms in ABCB1 tested in patients enrolled in the FOCUS Trial did not predict response to irinotecan in combination with FU either.[20] These data strongly suggest that other drug resistance mechanisms must play a dominant role in determining the response of metastatic CRC to irinotecan. However, to our knowledge there are no clinical trials that use proportional hazard models to determine the influence of $\mathrm{ABC}$-transporters on the irinotecan response.

A number of issues may have precluded us from finding a significant association between $A B C$ transporter expression and irinotecan response. First, tumour heterogeneity is an intrinsic problem when creating TMAs [21, 22] and this may influence any study seeking to associate drug resistance and survival data with expression of single or a few markers. Nevertheless, TMAs are a very useful high-throughput screening method with high concordance comparing to full slide analysis and are therefore very useful when analysing large numbers of tumours. [23] To minimize the heterogeneity problem, the TMA that was generated for the current study contained $2.0 \mathrm{~mm}$ cores of each tumour, considerably bigger cores than the standard of $0.6 \mathrm{~mm}$.[24]

Second, we have used a patient cohort in which irinotecan was used as second line therapy. First line chemotherapy may have influenced the expression levels of ABC-transporters. Indeed, in vitro experiments have shown that pre-treatment with 5-FU or oxaliplatin alters mRNA levels of ABCB1 and ABCG2.[25] Multidrug resistance may either be acquired as a consequence of drug-induced alterations in tumour biology $[26,27]$ or as a consequence of drug-induced selection of pre-existent drug-resistant clones.[28] In this study, about 20 percent of all tumours received prior adjuvant therapy. Alterations or selection of pre-existent drug-resistant clones in these tumours could have influenced the results of this study, even though prior adjuvant therapy was not identified as an independent predictor in the CAIRO study.[17] However, when excluding these patients from the analyses we could still not find any correlation between $\mathrm{ABC}$ transporter expression and PFS.

Third, the TMA only contained samples of the primary CRC of the patients in the CAIRO cohort. Patients with resectable metastatic disease were not included in the CAIRO trial, nor were biopsies taken during or after treatment precluding the analysis of $A B C$ transporter expression in the metastasis itself. Therefore, we have used primary tumour specimens to predict treatment efficacy in metastatic disease. By analysing mRNA levels in paired primary tumours and metastases we showed that there is a mediocre to poor concordance.

Although we cannot exclude that ABC transporter expression may relate to response to irinotecan, we conclude from the present study that immunohistochemical analysis of ABCB1 and/or ABCG2 expression in primary $C R C$ has no value in the selection of patients for irinotecan therapy in metastatic CRC.

\section{Supplementary Material}

Supplemental Figure 1.

http://www.jcancer.org/v06p1079s1.pdf

\section{Abbreviations}

ABC: ATP-binding cassette;

CRC: Colorectal cancer;

CRLM: Colorectal Liver Metastasis;

TMA: Tissue Micro Array;

PFS: Progression Free Survival.

\section{Acknowledgements}

Funding:

BLE - Dutch cancer society.

KT - PON foundation; Foundation "Vrienden UMC Utrecht".

\section{Competing Interests}

The authors have declared that no competing interest exists.

\section{References}

1. Koopman M, Punt CJ. Chemotherapy, which drugs and when. European journal of cancer. 2009; 45 Suppl 1: 50-6.

2. Alagoz M, Gilbert DC, El-Khamisy S, Chalmers AJ. DNA repair and resistance to topoisomerase I inhibitors: mechanisms, biomarkers and therapeutic targets. Current medicinal chemistry. 2012; 19: 3874-85.

3. Gilbert DC, Chalmers AJ, El-Khamisy SF. Topoisomerase I inhibition in colorectal cancer: biomarkers and therapeutic targets. British journal of cancer. 2012; 106: 18-24.

4. Emmink BL, Van Houdt WJ, Vries RG, Hoogwater FJ, Govaert KM, Verheem A, et al. Differentiated human colorectal cancer cells protect tumor-initiating cells from irinotecan. Gastroenterology. 2011; 141: 269-78.

5. te Velde EA, Vooten JM, Gebbink MF, van Gorp JM, Voest EE, Borel Rinkes $\mathrm{IH}$. Enhanced antitumour efficacy by combining conventional chemotherapy with angiostatin or endostatin in a liver metastasis model. The British journal of surgery. 2002; 89: 1302-9.

6. Gottesman MM, Fojo T, Bates SE. Multidrug resistance in cancer: role of ATP-dependent transporters. Nature reviews Cancer. 2002; 2: 48-58. 
7. Candeil L, Gourdier I, Peyron D, Vezzio N, Copois V, Bibeau F, et al. ABCG2 overexpression in colon cancer cells resistant to SN38 and in irinotecan-treated metastases. International journal of cancer Journal international du cancer. 2004; 109: 848-54.

8. Robey RW, Polgar O, Deeken J, To KW, Bates SE. ABCG2: determining its relevance in clinical drug resistance. Cancer metastasis reviews. 2007; 26: 39-57.

9. Kosinski C, Li VS, Chan AS, Zhang J, Ho C, Tsui WY, et al. Gene expression patterns of human colon tops and basal crypts and BMP antagonists as intestinal stem cell niche factors. Proceedings of the National Academy of Sciences of the United States of America. 2007; 104: 15418-23.

10. Calcagno AM, Ludwig JA, Fostel JM, Gottesman MM, Ambudkar SV. Comparison of drug transporter levels in normal colon, colon cancer, and Caco-2 cells: impact on drug disposition and discovery. Molecular pharmaceutics. 2006; 3: 87-93.

11. Maliepaard M, Scheffer GL, Faneyte IF, van Gastelen MA, Pijnenborg AC, Schinkel AH, et al. Subcellular localization and distribution of the breast cancer resistance protein transporter in normal human tissues. Cancer research. 2001; 61: 3458-64.

12. Thiebaut F, Tsuruo T, Hamada H, Gottesman MM, Pastan I, Willingham MC. Cellular localization of the multidrug-resistance gene product P-glycoprotein in normal human tissues. Proceedings of the National Academy of Sciences of the United States of America. 1987; 84: 7735-8.

13. Middleton G, Brown S, Lowe C, Maughan T, Gwyther S, Oliver A, et al. A randomised phase III trial of the pharmacokinetic biomodulation of irinotecan using oral ciclosporin in advanced colorectal cancer: results of the Panitumumab, Irinotecan \& Ciclosporin in COLOrectal cancer therapy trial (PICCOLO). European journal of cancer. 2013; 49: 3507-16.

14. Weroha SJ, Oberg AL, Ziegler KL, Dakhilm SR, Rowland KM, Hartmann LC, et al. Phase II trial of lapatinib and topotecan (LapTop) in patients with platinum-refractory/resistant ovarian and primary peritoneal carcinoma. Gynecologic oncology. 2011; 122: 116-20.

15. Linn SC, Giaccone G, van Diest PJ, Blokhuis WM, van der Valk P, van Kalken $\mathrm{CK}$, et al. Prognostic relevance of P-glycoprotein expression in breast cancer. Annals of oncology : official journal of the European Society for Medical Oncology / ESMO. 1995; 6: 679-85.

16. Shaffer BC, Gillet JP, Patel C, Baer MR, Bates SE, Gottesman MM. Drug resistance: still a daunting challenge to the successful treatment of AML. Drug resistance updates : reviews and commentaries in antimicrobial and anticancer chemotherapy. 2012; 15: 62-9.

17. Koopman M, Antonini NF, Douma J, Wals J, Honkoop AH, Erdkamp FL, et al. Sequential versus combination chemotherapy with capecitabine, irinotecan, and oxaliplatin in advanced colorectal cancer (CAIRO): a phase III randomised controlled trial. Lancet. 2007; 370: 135-42.

18. Koopman M, Kortman GA, Mekenkamp L, Ligtenberg MJ, Hoogerbrugge N, Antonini NF, et al. Deficient mismatch repair system in patients with sporadic advanced colorectal cancer. British journal of cancer. 2009; 100: 266-73.

19. Pizzini S, Bisognin A, Mandruzzato S, Biasiolo M, Facciolli A, Perilli L, et al. Impact of microRNAs on regulatory networks and pathways in human colorectal carcinogenesis and development of metastasis. BMC genomics. 2013; 14: 589.

20. Braun MS, Richman SD, Quirke P, Daly C, Adlard JW, Elliott F, et al. Predictive biomarkers of chemotherapy efficacy in colorectal cancer: results from the UK MRC FOCUS trial. Journal of clinical oncology : official journal of the American Society of Clinical Oncology. 2008; 26: 2690-8.

21. Berlth F, Monig SP, Schlosser HA, Maus M, Baltin CT, Urbanski A, et al. Validation of 2-mm tissue microarray technology in gastric cancer. Agreement of 2-mm TMAs and full sections for Glut-1 and Hif-1 alpha. Anticancer research. 2014; 34: 3313-20.

22. Goethals L, Perneel C, Debucquoy A, De Schutter H, Borghys D, Ectors N, et al. A new approach to the validation of tissue microarrays. The Journal of pathology. 2006; 208: 607-14.

23. Batistatou A, Televantou D, Bobos M, Eleftheraki AG, Kouvaras E, Chrisafi S, et al. Evaluation of current prognostic and predictive markers in breast cancer: a validation study of tissue microarrays. Anticancer research. 2013; 33: 2139-45.

24. Veenendaal LM, Kranenburg O, Smakman N, Klomp A, Borel Rinkes IH, van Diest PJ. Differential Notch and TGFbeta signaling in primary colorectal tumors and their corresponding metastases. Cellular oncology : the official journal of the International Society for Cellular Oncology. 2008; 30: 1-11.

25. Theile D, Grebhardt S, Haefeli WE, Weiss J. Involvement of drug transporters in the synergistic action of FOLFOX combination chemotherapy. Biochemical pharmacology. 2009; 78: 1366-73.

26. Wu Q, Yang Z, Nie Y, Shi Y, Fan D. Multi-drug resistance in cancer chemotherapeutics: mechanisms and lab approaches. Cancer letters. 2014; 347: 159-66.

27. Di Nicolantonio F, Mercer SJ, Knight LA, Gabriel FG, Whitehouse PA, Sharma S, et al. Cancer cell adaptation to chemotherapy. BMC cancer. 2005; 5: 78.

28. Gerlinger M, Rowan AJ, Horswell S, Larkin J, Endesfelder D, Gronroos E, et al. Intratumor heterogeneity and branched evolution revealed by multiregion sequencing. The New England journal of medicine. 2012; 366: 883-92. 